\title{
New method for the determination of benzoic and sorbic acids in commercial orange juices based on second-order spectrophotometric data generated by a $\mathrm{pH}$ gradient flow injection technique
}

(Supporting Information)

\author{
NILDA R. MARSILI,$^{\dagger}$ ADRIANA LISTA, ${ }^{\dagger}$ BEATRIZ S. FERNANDEZ BAND,, \\ HÉCTOR C. GOICOECHEA ${ }^{\dagger, \S, *}$ AND ALEJANDRO C. OLIVIERI ${ }^{\#, * *}$
}

Cátedra de Química Analítica, Facultad de Bioquímica y Ciencias Biológicas,

Universidad Nacional del Litoral, Ciudad Universitaria, Santa Fe S3000 CC. 242,

Argentina, Laboratorio FIA-Química Analítica, Departamento de Química, Universidad Nacional del Sur, Av. Alem 1253, (B8000CPB) Bahía Blanca, Argentina, Department of Chemistry and Molecular Biology, North Dakota State University, Fargo, ND 581055516, United States of America,

Departamento de Química Analítica, Facultad de Ciencias Bioquímicas y

Farmacéuticas, Universidad Nacional de Rosario, Suipacha 531, Rosario (S2002LRK)

Corresponding authors (hhgoico@ fbcb.unl.edu.ar, aolivier@fbioyf.unr.edu.ar, usband@ criba.edu.ar).

${ }^{\dagger}$ Cátedra de Química Analítica.

${ }^{\ddagger}$ Laboratorio FIA-Química Analítica.

${ }^{\S}$ Department of Chemistry and Molecular Biology

\# Departamento de Química Analítica 


\section{THEORY}

PARAFAC. When a sample produces a $J \times K$ data matrix, such as the $\mathrm{pH}$-spectral landscape $(J=$ number of absorption wavelengths, $K=$ number of $\mathrm{pH}$ intervals $)$, the corresponding set obtained by 'stacking' the training matrices is a three-dimensional array, of dimensions $I \times J \times K(I=$ number of samples, see Fig. 1, top). Provided the data follow a trilinear model, the array can be written as a sum of Kronecker (1) products of three vectors for each absorbent component. If $\mathbf{a}_{n}, \mathbf{b}_{n}$ and $\mathbf{c}_{n}$ collect the relative concentration $(I \times 1)$, absorption $(J \times 1)$ and $\mathrm{pH}(K \times 1)$ profiles for component $n$ respectively, the array of data $\underline{\mathbf{F}}$ can be written as $(1,2)$ :

$$
\underline{\mathbf{F}}=\sum_{n=1}^{N} \mathbf{a}_{n} \otimes \mathbf{b}_{n} \otimes \mathbf{c}_{n}+\underline{\mathbf{E}}
$$

where $\otimes$ indicates Kronecker product, $N$ is the total number of responsive components and $\underline{\mathbf{E}}$ is a residual error term of the same dimensions as $\underline{\mathbf{F}}$. The column vectors $\mathbf{a}_{n}, \mathbf{b}_{n}$ and $\mathbf{c}_{n}$ are usually collected into the three loading matrices $\mathbf{A}, \mathbf{B}$ and $\mathbf{C}$. Decomposition of the three-way array provides the profiles (B and $\mathbf{C}$ ) and relative concentrations (A) of individual components in the $I$ mixtures, whether they are chemically known or not. This constitutes the basis of the so-called second-order advantage. Theoretically, this property should allow the analyst to obtain the concentration values of calibrated constituents in the presence of any number of uncalibrated components.

PARAFAC is becoming more and more employed by chemometricians and analytical spectroscopists for analyzing three-way data, and the software required for its implementation is easily available on the internet (3). Issues relevant to the application of the model are: 1) how to establish the number of components, 2) how to identify specific components from the information provided by the array decomposition and 3) 
how to calibrate the model in order to obtain absolute concentrations for a particular component in an unknown sample.

The number of responsive components $(N)$ can be estimated by several different methods. The consideration of the PARAFAC internal parameter known as core consistency (4) and the study of the regression error of the pseudounivariate calibration line (5) are useful techniques. In the present case, where relevant information concerning the properties of the system is needed, the number of components is equal to the number of different species in the sample. For synthetic binary mixtures, this number is four, on account of the presence of benzoic acid, benzoate, sorbic acid and sorbate in equilibrium. For real samples the number is likely to be larger, since a background arising from the fruit juice is also present, and consists of the sum of the spectra for the several juice components, which may also be $\mathrm{pH}$ dependent. However, satisfactory results were obtained by including a single interferent component, which was considered as an average background effect.

Identification of the chemical constituent under investigation is done with the aid of the profiles $\mathbf{B}$ and $\mathbf{C}$, as extracted by PARAFAC, and comparing them with those for a standard solution of the analyte of interest.

Absolute analyte concentrations are obtained after proper calibration, since only relative values (A) are provided by decomposing the three-way data array. Experimentally, this is done by using the information for the set of standards of known composition. The calibration procedure involves decomposing an array formed by joining the matrices for the $I$ training samples with that for the unknown. In the presently studied case, each analyte has two species at equilibrium, hence PARAFAC will provide two concentration values, which should ideally be equal. Averaging both values provides an estimation of the analyte concentration in the unknown. It should be 
noticed that employment of this latter mode implies that the array decomposition should be repeated for each newly analyzed sample. Table 1 summarizes the entire procedure.

It should be noticed that PARAFAC initialization is conveniently performed in the present case by supplying the model with initial guesses of $\mathbf{A}, \mathbf{B}$ and $\mathbf{C}$. The $\mathbf{B}$ profiles are initially set as equal to spectra of the analytes in their acid and basic forms, and, for real samples, including a spectrum of a pool of different juices (all normalized to unit length). The $\mathbf{C}$ profiles are normalized squared-pulse functions for each analyte species, and a constant function for the background juice in real sample applications. Finally, an approximation to the A profile is given in terms of: 1) the known concentration of the analytes in the calibration samples, 2) mean calibration concentrations for the analytes in the unknown, and 3) a single value for the background juice, obtained after normalizing its spectrum.

MCR-ALS. MCR-ALS is a method capable of dealing with data sets deviating from trilinearity. Instead of forming a three-dimensional data array, the latter is unfolded along the mode which is suspected of breaking the trilinear structure, i.e. if a matrix-tomatrix variation of profiles occurs along the column direction, a column-wise augmented matrix is created (see Fig. 1, bottom). The bilinear decomposition of the augmented matrix $\mathbf{D}$ is performed according to the expression:

$$
\mathbf{D}=\mathbf{G} \times \mathbf{S}^{\mathrm{T}}+\mathbf{E}
$$

where the rows of $\mathbf{D}$ contain the absorption spectra measured as a function of $\mathrm{pH}$, the columns of $\mathbf{G}$ contain the $\mathrm{pH}$ profiles of the intervening species, the columns of $\mathbf{S}$ their related spectra, and $\mathbf{E}$ is a matrix of residuals not fitted by the model. Appropriate dimensions of $\mathbf{D}, \mathbf{G}, \mathbf{S}$ and $\mathbf{E}$ are thus $(1+I) \times J,(1+I) \times N, J \times N$ and $(1+I) \times J$ respectively ( $I, J, K$ and $N$ have the same meaning as for PARAFAC). Decomposition of $\mathbf{D}$ is achieved by iterative least-squares minimization of $\|\mathbf{E}\|$ under suitable 
constraining conditions, namely nonnegativity in spectral profiles, unimodality (and nonnegativity) in concentration profiles, and also closure relations between acid-base pairs of a given component. The pure spectra of the compounds should be the same in all experiments, but, in contrast to PARAFAC, the profiles in the different $\mathbf{G}$ submatrices need not share a common shape. This is the reason why experiments performed in different conditions (e.g. temperature, slight $\mathrm{pH}$ mismatches, etc.) and, hence, showing different $\mathrm{pH}$ profiles, can be analyzed together as long as the spectra of the compounds involved in the process remain invariant (absorption spectra usually show a limited temperature dependence).

It is necessary to point out, in the present context, that MCR-ALS does also require initialization with system parameters as close as possible to the final results. In the column-wise augmentation mode, the species spectra are required, as obtained from either pure analyte standards or from the analysis of purest spectra (6). In the case of truly unknown samples with possible interferents, we have found that successful application of MCR-ALS to our case required the combination of both of the above procedures, i.e., known spectra for pure analytes and purest spectral analysis for the interferent.

As with PARAFAC, calibration is possible when the unknown sample data matrix is augmented with matrices obtained for standards. Usually, a limited number of standards is employed $(7,8)$. In order to properly compare the results furnished by MCR-ALS with those of PARAFAC, however, we chose to augment the sample data matrix with the matrices for all the nine calibration mixtures. After decomposition of $\mathbf{D}$, concentration information contained in $\mathbf{C}$ can be used for quantitative predictions in a manner similar to that described above for PARAFAC, i.e., by building a pseudounivariate calibration graph with the relative concentrations of the training samples 
contained in the optimized matrix $\mathbf{C}$, and subsequent interpolation of the value for the unknown sample (see Table 1 for a summary of the MCR-ALS procedure).

$N$-PLS. Multiway regression methods such as $N$-PLS extend the traditional PLS algorithm to higher orders, using the multidimensional structure of the data for model building and prediction (9). In the case of three-way data, the model is given by the following equation:

$$
x_{i j k}=\sum_{f=1}^{F} t_{t f} w_{j f}^{J} w_{k f}^{K}+e_{i j k}
$$

where $x_{i j k}$ is the absorbance measured for sample $i$ at wavelength $j$ and $\mathrm{pH} k, F$ is the number of components, $t_{t f}$ is an element of the score matrix $\mathbf{T}, w_{j f}^{J}$ and $w_{k f}^{K}$ are elements of two $\mathbf{W}$ loading matrices, and $e_{i j k}$ is a residue not fitted by the model. The model finds the scores yielding maximum covariance with analyte concentrations as the dependent variable, in a three-dimensional sense. The advantage of using N-PLS over bidimensional regression is a stabilization of the decomposition involved in eq. (3), which potentially gives increased interpretability and better predictions. However, this methodology does not exploit the second-order advantage, and in principle, it should lead to results of a lower quality as compared to PARAFAC or MCR-ALS in samples of complex composition. 


\section{LITERATURE CITED}

(1) Ewing, G.W. Instrumental methods of chemical analysis, McGraw-Hill, New York, 1985.

(2) Leurgans, S.; Ross, R.T. Multilinear models in spectroscopy. Statist. Sci., 1992, 7, 289-319.

(3) http://www.models.kvl.dk/source/

(4) Bro, R. Multi-way analysis in the food industry, Doctoral thesis, University of Amsterdam, Netherlands, 1998.

(5) Muñoz de la Peña, A.; Espinosa Mansilla, A.; González Gómez, D.; Olivieri, A.C.; Goicoechea, H.C. Interference-free analysis using three-way fluorescence data and the parallel factor model. Determination of fluoroquinolone antibiotics in human serum. Anal. Chem. 2003, 75, 2640-2646.

(6) Windig, W.; Guilment, J. Interactive self-modeling mixture analysis. Anal. Chem., 1991, 63, 1425-1432.

(7) Vives, M.; Gargallo, R.; Tauler, R. Study of the Intercalation Equilibrium between the Polynucleotide Poly(adenylic)-Poly(uridylic) Acid and the Ethidium Bromide Dye by Means of Multivariate Curve Resolution and the Multivariate Extension of the Continuous Variation and Mole Ratio Methods. Anal. Chem. 1999, 71, 4328-4337

(8) Smilde, A.K.; Tauler, R.; Saurina, J.; Bro, R. Calibration methods for complex second-order data. Anal. Chim. Acta 1999, 398, 237-251

(9) Bro, R. Multiway calibration. Multilinear PLS. J. Chemometrics, 1996, 10, $47-$ 61. 
Table 1. Stepwise description of PARAFAC and MCR-ALS calibrations for the presently studied three-way data

\begin{tabular}{|c|c|c|}
\hline Step & PARAFAC & MCR-ALS \\
\hline 1 & $\begin{array}{l}\text { Build an }(I+1) \times J \times K \text { three-way } \\
\text { array with the first } I \text { spectral-pH } \\
\text { evolutions for the training samples } \\
\text { and the last one for the unknown }\end{array}$ & $\begin{array}{l}\text { Build an }(I+1) \times J \times K \text { augmented } \\
\text { matrix with the first I spectral-pH } \\
\text { evolutions for the training samples } \\
\text { and the last one for the unknown }\end{array}$ \\
\hline 2 & $\begin{array}{l}\text { Supply initial guesses for A, B and } \\
\text { C profiles. }\end{array}$ & Supply initial guesses for $\mathbf{S}$ and $\mathbf{G}$. \\
\hline 3 & $\begin{array}{l}\text { Decompose the array and obtain } \\
\mathbf{A}, \mathbf{B} \text { and } \mathbf{C} \text {, imposing non- } \\
\text { negativity in all modes. }\end{array}$ & $\begin{array}{l}\text { Perform alternating least squares } \\
\text { fit to obtain } \mathbf{S} \text { and } \mathbf{G} \text {, imposing non- } \\
\text { negativity in all modes. }\end{array}$ \\
\hline 4 & $\begin{array}{l}\text { Identify from } \mathbf{B} \text { and } \mathbf{C} \text { the pair of } \\
\text { profiles corresponding to both } \\
\text { species of the analyte of interest } \\
\left(n_{1} \text { and } n_{2}\right)\end{array}$ & $\begin{array}{l}\text { Identify from } \mathbf{S} \text { the pair of profiles } \\
\text { corresponding to both species of } \\
\text { the analyte of interest }\left(n_{1} \text { and } n_{2}\right)\end{array}$ \\
\hline \multirow[t]{3}{*}{5} & $\begin{array}{l}\text { Separately regress the first } I \\
\text { elements of columns } \mathbf{a}_{n 1} \text { and } \mathbf{a}_{n 2} \\
\text { against known standard analyte } \\
\text { concentrations } \mathbf{c}_{\text {cal }} \text { (pseudo- } \\
\text { univariate calibration), and obtain } \\
k_{1} \text { and } k_{2} \text { : }\end{array}$ & $\begin{array}{l}\text { Separately regress the first } / \\
\text { elements of columns } \mathbf{g}_{n 1} \text { and } \mathbf{g}_{n 2} \\
\text { against known standard analyte } \\
\text { concentrations } \mathbf{c}_{\text {cal }} \text { (pseudo- } \\
\text { univariate calibration), and obtain } \\
k_{1} \text { and } k_{2} \text { : }\end{array}$ \\
\hline & {$\left[\begin{array}{lll}a_{1, n 1} & \ldots & a_{l, n 1}\end{array}\right]=k_{1} \times \mathbf{c}_{\mathrm{cal}}^{\top}$} & {$\left[g_{1, n 1} \ldots g_{l, n 1}\right]=k_{1} \times \mathbf{c}_{\mathrm{cal}}^{\top}$} \\
\hline & {$\left[\begin{array}{lll}a_{1, n 2} & \ldots & a_{1, n 2}\end{array}\right]=k_{2} \times \mathbf{c}_{\mathrm{cal}}^{\top}$} & {$\left[g_{1, n 2} \ldots g_{l, n 2}\right]=k_{2} \times \mathbf{c}_{\mathrm{cal}}^{\top}$} \\
\hline \multirow[t]{3}{*}{6} & $\begin{array}{l}\text { Convert relative to absolute } \\
\text { concentration in the unknown, } \\
\text { starting from the last elements of } \\
\text { columns } \mathbf{a}_{n 1} \text { and } \mathbf{a}_{n 2} \text { : }\end{array}$ & $\begin{array}{l}\text { Convert relative to absolute } \\
\text { concentration in the unknown, } \\
\text { starting from the last elements of } \\
\text { columns } \mathbf{g}_{n 1} \text { and } \mathbf{g}_{n 2} \text { : }\end{array}$ \\
\hline & $c_{\text {unk }, 1}=a_{l+1, n 1} / k_{1}$ & $c_{\text {unk }, 1}=g_{l+1, n 1} / k_{1}$ \\
\hline & $c_{\text {unk }, 2}=a_{1+1, n 2} / k_{2}$ & $C_{\text {unk }, 2}=g_{l+1, n 2} / k_{2}$ \\
\hline \multirow[t]{2}{*}{7} & $\begin{array}{l}\text { Average the results obtained for } n_{1} \\
\text { and } n_{2} \text { : }\end{array}$ & $\begin{array}{l}\text { Average the results obtained for } n_{1} \\
\text { and } n_{2} \text { : }\end{array}$ \\
\hline & $c_{\text {unk }}=\left(c_{\text {unk }, 1}+c_{\text {unk }, 2}\right) / 2$ & $c_{\text {unk }}=\left(c_{\text {unk }, 1}+c_{\text {unk }, 2}\right) / 2$ \\
\hline
\end{tabular}




\section{FIGURE CAPTIONS}

Figure 1. Top: stacking of the unknown sample data matrix and the training samples to form a three-way array for PARAFAC study, showing the relevant array dimensions. Bottom: matrix augmentation of the sample data matrix with the training samples for MCR-ALS analysis. 


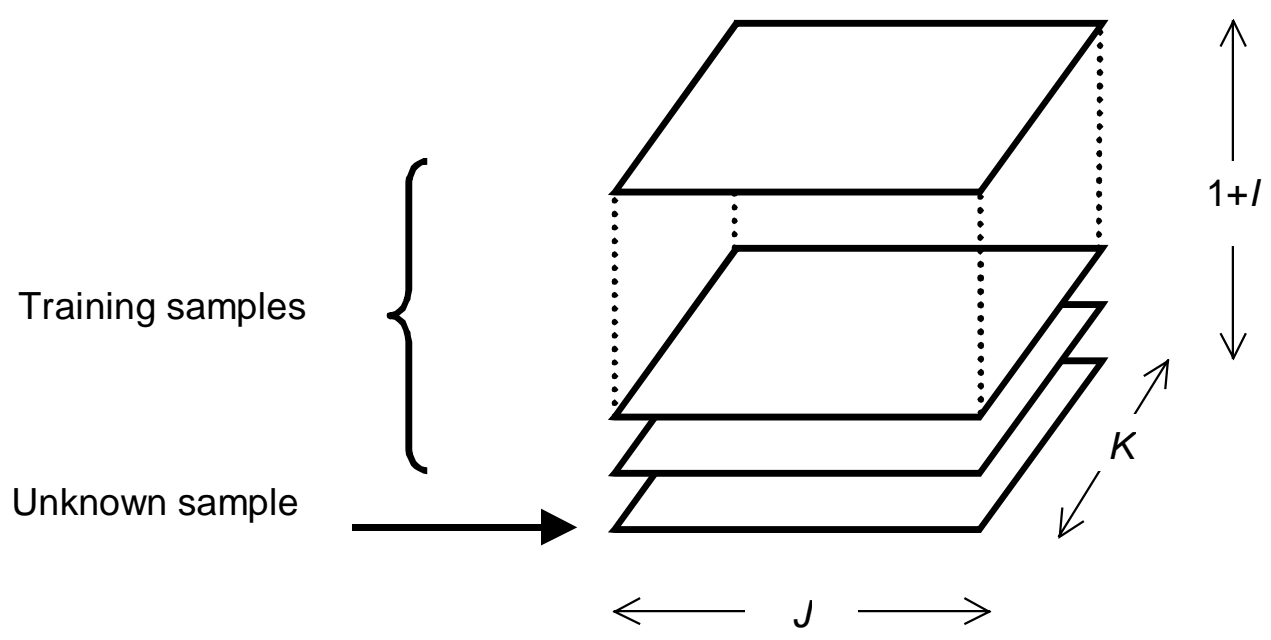

Training samples

Unknown sample

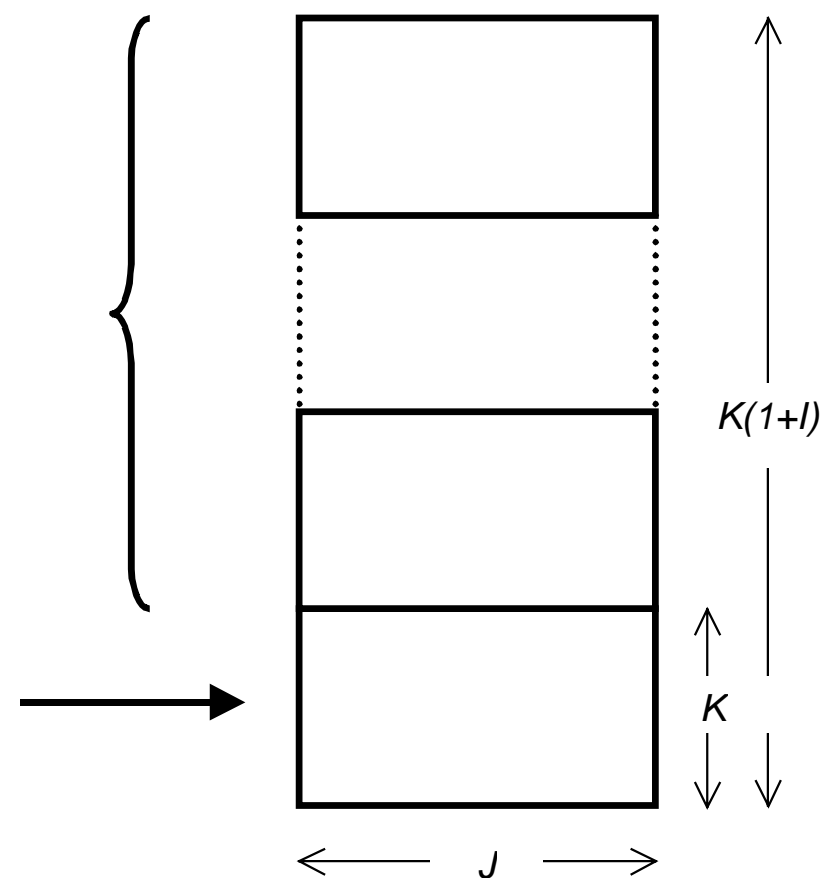

Figure 1 